\title{
Efecto de distintos planteamientos metodológicos en clases de educación física sobre el aprendizaje de tres habilidades de voleibol
}

\section{Effect of the application of three different methodological approaches on the learning of three techniques of volleyball in physical education}

\author{
Elena Hernández-Hernández ${ }^{1 *}$, Enrique Ortega Toro², Manuel Mayordomo ${ }^{3}$ y José Manuel Palao Andrés ${ }^{4}$ \\ 1 Universidad Pablo de Olavide, Departamento de deporte e informática. Sevilla (España). 2 Universidad de Murcia, Facultad de Ciencias del Deporte, \\ Campus de San Javier. Murcia (España). 3 Licenciado en Actividad Fisica y deporte. 4 University of Wisconsin (Parkside), Health, Exercise Science and Sport \\ Management, Somers (United State).
}

Resumen: El objetivo del presente trabajo fue conocer el efecto de la aplicación de planteamientos metodológicos diferentes sobre el aprendizaje de tres gestos técnicos durante una unidad didáctica de voleibol en clase de educación física. La muestra utilizada fue de 68 alumnos de tres clases de $1^{\circ}$ curso de Educación Secundaria Obligatoria. Se llevó a cabo un diseño cuasi-experimental intergrupo con pre y post-test, con un tratamiento de seis sesiones. La variable independiente fue la forma de organizar la clase en función del planteamiento metodológico. Las variables dependientes fueron: a) el aprendizaje de las habilidades a nivel cualitativo y cuantitativo; b) el grado de conocimiento teóricopráctico adquiridos sobre técnica y táctica tras la aplicación de las sesiones; y c) la calidad y cantidad de práctica realizada; d) la percepción del alumno y e) la percepción del profesor. Los resultados indican que cada planteamiento metodológico (tradicional, comprensivo y educción deportiva) presenta ventajas e inconvenientes. Su empleo variará en fun- ción de las características y necesidades del grupo con el que el docente se encuentre

Palabras clave: modelo de enseñanza, deporte, iniciación, percepción, alumno.

Abstract: The aim of this study is to assess the effect of the application of three different methodological approaches on the learning of three techniques used in volleyball. A sample of 68 Spanish students aged 13-14 was analyzed according to a quasi-experimental inter-group design with pre- and post-tests during six sessions. The independent variable was the way of organizing the class. The dependent variables were: a) the learning of the skills; b) knowledge; c) quality and quantity of practice; d) student's perception; and e) teacher's perception. The results show that each approach (traditional, comprehensive and sport education) has benefits and drawbacks. Their implementation depends on the features and needs of the group led by the professor. Key words: teaching, sport, sport initiation, perception, student.

\section{Introducción}

En los últimos años, son numerosos los trabajos que tratan de explicar el por qué del descenso del nivel de implicación de los alumnos, en las clases de educación física. Algunos expertos en la temática (García-López, Gutiérrez-Díaz, González-Víllora y Valero, 2012; González-Víllora, García-López, Contreras-Jordán y Sánchez-Mora, 2009 ; Kirk, 2006; Mitchell, Oslin y Griffin, 2006; Navarro, 2004; Serra-Olivares, J., 2014; Siedentop, Hastie, y Van der Mars, 2011) han tratado de buscar soluciones con propuestas como: a) el empleo de modelos de enseńanza comprensivos (siempre que el control de la clase lo permita); b) la reducción del uso de las vivencias competitivas; la introducción de los contenidos a través de juegos (búsqueda del componente lúdico y de diversión dentro de las clases); c) el papel activo del alumno en el proceso de adquisición de su aprendizaje; y d) la diversidad de los contenidos enseńados a lo largo del curso. Con el objeto de incrementar esos niveles de implicación y motivación por

Dirección para correspondencia [Correspodence address]: Elena Hernández Hernández, Universidad Pablo de Olavide, Departamento de deporte e informática, ctra. de Utrera, km. 1. 41013. Sevilla (España). E-mail: ehernandez@upo.es las sesiones en el entorno escolar, se han diseñado trabajos de investigación en los que se utilizan diferentes planteamientos metodológicos. Algunos ejemplos, son los trabajos desarrollados por Calderón, Martínez y Hastie (2013), donde se compara la motivación, la implicación y el aprendizaje de alumnos y profesores al implementar un modelo de enseńanza tradicional y otro más innovador, como el modelo de educación deportiva, en una muestra de primaria. Otros como los de Méndez-Giménez, Fernández-Río, Méndez-Alonso (2015), comparan el efecto de ambos modelos sobre la deportividad y motivación en una muestra de casi 300 estudiantes entre educación secundaria y bachiller. En otros, como los de Gutiérrez-Díaz, García-López, Chaparro, Fernández (2014), se incluye, además, como estrategia metodológica, un modelo comprensivo como el Teaching Games for Understanding. En un trabajo de revisión, Evangelio, González-Villora, Serra-Olivares, y Pastor-Vicedo (2016) analizan los estudios publicados en Espańa, entre los años 2010-2014, donde se ha aplicado el MED en contextos reglados. En todos ellos, se observan mejores resultados al aplicar el MED respecto a metodologías más tradicionales, en lo referente a aspectos como la motivación, implicación en clase, y otros valores so- 
ciales. Sin embargo, hay pocos estudios donde, además, se proporcione información del efecto de estos planteamientos metodológicos en la adquisición, o no, de otro tipo de contenidos de carácter más procedimental y/o conceptual.

Con la finalidad de aportar alguna respuesta a la cuestión anterior, el presente trabajo estudia el efecto de organizar la clase de educación física siguiendo tres planteamientos metodológicos diferentes. El estudio pretende comprobar las bondades que pueden aportar los diferentes modelos escogidos, en busca de fórmulas metodológicas que faciliten la adquisición de diferentes tipos de contenidos, junto con el incrementen en los niveles de motivación hacia la práctica y el éxito, a través de la autonomía y participación activa del alumno. Para ello se han seleccionado tres planteamientos:

1) Planteamiento metodológico basado en un modelo tradicional, donde el profesor tiene el control de la situación, y el aprendizaje se busca a través de tareas (MT). Es un modelo que toma su base en la tecnificación y mecanización de habilidades motrices específicas (González-Víllora, et al., 2009). Para el diseño de las sesiones y el de las tareas se siguió un enfoque conductista (Kirk y Macdonald, 1998; Light, 2008), donde la responsabilidad de las clases recae enteramente en la figura del profesor.

2) Planteamiento metodológico basado en un modelo más comprensivo, donde el profesor tiene el control de la situación y el aprendizaje se busca a través de formas jugadas o tareas aplicadas (MC). En ellas se utiliza el juego como elemento facilitador de la participación del alumno en clase, y de adaptación de la tarea a contextos de juego más reales (Gutiérrez-Díaz at al., 2014). Para el diseño de las tareas se realizó una adaptación del modelo "Teaching Games For Understanding” (Griffin y Butler, 2005).

3) Planteamiento metodológico basado en el modelo de educación deportiva donde el alumno tiene el control de la situación y participa de forma activa (MED). La ED se basa en una pedagogía cooperativa y constructivista, que se ve facilitada por el trabajo en pequeños grupos y juegos reducidos, en los que cada miembro tiene un rol y, en general, se persigue un objetivo de mejora técnica, conceptual, mejora en la toma de decisiones y mejora en el grado de responsabilidad y autonomía (Calderón, Martínez de Ojeda \& Hastie, 2013; Martínez de Ojeda, Calderón \& Campos, 2012). Para el diseño de las sesiones y tareas se realizó una adaptación del modelo "Sport Education" (Siedentop, Hastie, y Van der Mars, 2004).

De esta forma, el propósito de este estudio fue examinar la relación que hay entre tres modelos de enseñanza diferentes (MT, MC Y MED) sobre el aprendizaje y conocimiento de tres gestos técnicos, y la calidad / cantidad de práctica realizada durante la aplicación de una unidad didáctica de voleibol en un entorno educativo reglado.

\section{Método}

\section{Muestra}

Para el estudio se utilizó una muestra intencional compuesta por 67 alumnos de tres clases de un colegio de Enseñanza Secundaria del sureste de España (25, 19, y 24 alumnos por clase, respectivamente). Este centro es público, y está localizado en el municipio de Alguazas (Murcia). En el centro conviven población de clase media, trabajadores y población proveniente de la inmigración. Los alumnos estaban en primer curso de Enseñanza Secundaria (media: 13.7 años). Las clases fueron impartidas por una profesora licenciada en ciencias de la actividad física y del deporte que tenía 10 años de experiencia y entrenadora nacional de voleibol. Previamente se obtuvo los consentimientos informados de los padres, la asociación de padres y madres, del departamento de educación, y de la dirección del centro.

Las pautas que se tuvieron en cuenta para la selección del centro fueron la accesibilidad al mismo, y la disponibilidad de la muestra. Previo al estudio se les pasó a todos los participantes un cuestionario acerca de la actividad física deportiva que realizaban, con objeto de excluir a aquellos que tuvieran alguna patología limitante o hubieran tenido alguna experiencia previa en el deporte practicado en la unidad didáctica (voleibol). Los criterios de inclusión fueron: a) que los estudiantes perteneciesen a primer curso de la ESO en el centro escolar en el que se llevó a cabo la investigación; y b) que su edad cronológica correspondiese con la del curso en la que cursaban, no teniéndose en cuenta a los alumnos repetidores. Los criterios de exclusión fueron: a) no asistir a dos sesiones; b) no realizar el pre-test, o el pos-test; o c) realizar o haber practicado como actividad extraescolar voleibol.

\section{Diseño}

Se realizó un diseño cuasi-experimental, con pre-test y posttest y con un tratamiento de seis sesiones de 50 minutos (dos sesiones por semana). Las valoraciones se realización en la primera sesión y en la última sesión. La variable independiente fue el modelo de enseńanza aplicado por el docente en la unidad didáctica: a) Modelo tradicional (MT), donde el diseño de la unidad didáctica y el control de la clase eran dirigidas por el profesor a través de tareas o ejercicios; b) Modelo comprensivo (MC), donde el diseño de la unidad didáctica, y el control de la clase eran dirigidas por el profesor a través de formas jugadas (o situaciones de juego reducidas); y c) Modelo de educación deportiva (MED), donde el diseño de la 
unidad didáctica fu realizado por el profesor, y el control de la clase por los alumnos, a través de tareas/formas jugadas.

Las variables dependientes fueron: a) el aprendizaje de las habilidades a nivel cualitativo (control técnico sobre el balón) y a nivel cuantitativo (número de aciertos); b) el conocimiento adquirido; c) calidad y cantidad de practica; d) percepción del alumno, y e) percepción del profesor. Las variables de control fueron: a) experiencias previas deportivas; b) practica de actividad física fuera de clases de educación física, c) asistencia a clase; y d) existencia de lesiones u otro factor limitante a nivel físico.

\section{Procedimiento}

Antes de iniciar la aplicación de la unidad didáctica, la profesora realizó una serie de reuniones de orientación. En ellas se establecieron las tareas y los test a realizar en la unidad didáctica, los objetivos, los aspectos claves a lograr a nivel técnico, y la dinámica y los aspectos prácticos de las clases en los distintos planteamientos. Tras la orientación, la profesora aplicó los distintos planteamientos a tres líneas de primer curso de enseñanza secundaria.

La unidad didáctica consistió en la realización de ocho sesiones (tratamiento de seis sesiones) en las cuales se trabajó tres gestos técnicos de voleibol (saque de abajo, pase de dedos, y pase de antebrazos). Todas las clases tuvieron la misma estructura: calentamiento, explicación de la sesión (objetivos y actividades), parte principal y conclusión; y vuelta a la calma. El establecimiento de qué planteamiento iba a realizar cada grupo se realizó de forma aleatoria.

La información inicial fue aportada por el profesor en las clases controladas por el profesor (MT y MC). En el planteamiento donde el control de la clase lo ejercían los alumnos (MED), en la primera clase el profesor explicó la dinámica de trabajo, las funciones, la distribución de la clase en grupos, etc. El resto de sesiones fueron dirigidas por el entrenador/ capitán de cada equipo.

La información sobre el efecto del tratamiento fue valorada en tres momentos. Primera sesión, se realizó el pre-test (nivel de partida de los alumnos), durante las sesiones se valoró la calidad de las repeticiones, y el número de repeticiones, y al final de la unidad didáctica se valoró el aprendizaje logrado. En los tres modelos de enseńanza realizados, las tareas llevadas a cabo eran similares en objetivos, forma de realización, y tiempo de práctica. Las tareas se determinaron entre los investigadores y la profesora tomando como punto de partida para su diseño varios manuales sobre iniciación al deporte del voleibol (Palao y Hernández, 2007, 2009; Santos, Delgado, y Viciana, 1996).

En el MT, el docente era el encargado de dirigir la sesión, controlarla, y dar el feedback a los alumnos. En el MC, el profesor establecía la realización de formas jugadas, tanto cooperativa cómo competitivas (de 1 con 1 ó contra 1, a 3 con 3 o contra 3), su duración, y características. El control de la ejecución fue establecido por reglas de provocación, y supervisadas por el profesor. Las reglas de provocación son aquellas cuyo cumplimento favorecen de forma especial algunos comportamientos del alumno (Palao y Hernández, 2007) que, en este caso, iban destinadas a favorecer la correcta ejecución técnica de cada gesto. Estas reglas eran indicadas por el profesor al inicio y durante la sesión, y a través de pósters ubicados en el gimnasio. Estas reglas se añadieron a las reglas normales del voleibol. En el MED, la unidad didáctica era organizada por el profesor, si bien, se organizó la clase en equipos, y dentro de éstos, se establecieron funciones (entrenador/capitán, y jugador de campo). Las funciones cambiaban en cada sesión. Cada equipo era independiente del resto. En cada sesión tenía un tiempo de entrenamiento (tareas) y otro tiempo de partido (formas jugadas). Las actividades a realizar, sus características, y el tiempo de realización era marcado por el profesor, pero eran los entrenadores/capitanes los que explicaban y controlaban en cada equipo. Cada equipo tenía la sesión redactada y los aspectos claves de cada gesto. Los equipos trabajan de forma autónoma. El profesor era una ayuda para todos los equipos en caso de duda o aspecto que pudiese conllevar peligro.

El aprendizaje de las habilidades a nivel cualitativo, y el control técnico se valoró mediante el análisis observacional de la ejecución de cada intento, para ver si la acción era o no correcta. Los aspectos claves se establecieron a partir del análisis de la bibliografía específica (Palao y Hernández, 2007). Un panel de dos expertos (profesores universitarios con experiencia de más de siete años y entrenadores nacionales de voleibol) de forma conjunta con la profesora y los investigadores revisaron y concretaron los aspectos claves del gesto que debía cumplirse en cada gesto para cada intento. Los aspectos claves evaluados para el saque fueron: a) contactar el balón con una mano; b) lanzar el balón antes de golpearlo, c) lanzar el balón en frente del brazo de golpeo, y d) realizar la cadena cinética. Para el pase de dedos: a) contactar el balón con dos manos, b) contactar con el balón por encima de los hombros, c) contactar con el balón delante del cuerpo, y d) no acompañar el balón. Para el pase de antebrazos: a) contactar el balón con los dos brazos estirados, b) contactar con el balón por debajo de los hombros, c) contactar con el balón delante del cuerpo, y d) impulsar el balón con la acción de todo el cuerpo (cadena cinética). La observación fue realizada por un observador (licenciado en ciencias de la actividad física y deporte, con formación específica en voleibol). Se siguió el proceso de entrenamiento del observador definido por Behar (1993). La concordancia intra-observador fue de 0.98 o superior a todas las variables.

El aprendizaje de las habilidades a nivel cuantitativo fue valorado mediante el control del balón (grado de precisión). 
La evaluación se realizó por medio de una actividad en la que se trataba de enviar el balón a una diana tras superar la red. La distancia del alumno a la diana fue de $3 \mathrm{~m}$. Se registró el número de aciertos a partir del análisis de las filmaciones de las sesiones. Se realizaron un total de 3 intentos por gesto técnico

El aprendizaje conceptual se midió por medio de un cuestionario, el cuál valoró el grado de conocimientos teóricoprácticos acerca de la técnica y la táctica adquiridos por el alumno tras la aplicación de las sesiones (Hernández-Hernández y Palao, 2013). El cuestionario administrado constó de 20 preguntas cerradas, con cuatro posibles soluciones de aspectos conceptuales del voleibol (técnica, táctica, y reglamento).

La calidad y cantidad de práctica se obtuvo del análisis de los videos de las sesiones. Todos los contactos con el balón realizados por los alumnos en las sesiones fueron analizados $(\mathrm{n}=10.759)$. El cumplimiento de todos los objetivos marcados se entendió como la ejecución correcta. Se consideró como calidad el número de aspectos claves del gesto que cumplía el alumno en cada ejecución. Se entendió cómo cantidad el número de contactos con el balón por tarea. El análisis de las sesiones fue realizado por el mismo observador que realizó el análisis del aprendizaje procedimental.

Para la variable percepción del alumno, se tuvo en cuenta la valoración de cada alumno sobre cuanto creían ellos que habían aprendido en clase (dentro de una escala establecida por el instrumento de medida: mucho, poco, algo), y lo mismo en relación a lo que se habían divertido en clase. Para ello, se utilizó un instrumento validado (Palao y Hernández, 2012), que consistía en un cuestionario con dos preguntas. El instrumento seguía la estructura de un semáforo, ante las cuestiones: ¿Cuánto crees que has aprendido / te has divertido en esta sesión?, los alumnos debían responder con su opinión acerca de la clase realizada, escogiendo entre tres opciones identificadas con los colores rojo (poco), amarillo (algo), y verde (mucho). La sencillez del instrumento facilitó que los alumnos verbalizasen su percepción sobre lo que habían vivenciado en clase, a nivel de mejora en el nivel de las habilidades (aprendizaje) y diversión. Los cuestionarios se pasaron al final de cada una de las sesiones de tratamiento.

La percepción del profesor, de las condiciones de enseñanza evaluadas, fue medida a través de un diario y una entrevista semiestructurada al final de la unidad didáctica. El diario fue cumplimentado después de cada clase. En él se solicitaba que el profesor escribiera sus impresiones sobre los distintos protocolos, su influencia sobre sus funciones, además de su percepción acerca de la implicación de los alumnos en clase. La finalidad de la entrevista fue abordar aspectos relacionados con el efecto de la aplicación de los diferentes modelos de enseñanza, su opinión sobre cómo se había llevado a cabo el proceso de enseñanza-aprendizaje, la participación de los alumnos, y una evaluación global.

\section{Análisis de datos}

Para el análisis estadístico de los datos se utilizó el programa de análisis de datos software SPSS (ver. 17.0 para Microsoft Windows). La media y la desviación estándar de cada una de las tres condiciones de enseñanza fueron calculadas para el aprendizaje de las habilidades a nivel cualitativo y cuantitativo, el conocimiento, y la calidad y cantidad de práctica, la percepción del alumno y la percepción del profesor. Para valorar el aprendizaje se empleó un análisis de varianza (ANOVAs) tres (tipo unidad didáctica) x dos (momento de medición). Se utilizó el Test de Bonferroni para realizar las comparaciones Post Hoc. En todos los casos se utilizó un nivel de significación de $\mathrm{p}<.05$. Para el cálculo del poder estadístico se utilizó $\mathrm{h}^{2}$.

\section{Resultados}

\section{Aprendizaje de las habilidades}

Los resultados de las pruebas de control cualitativas de la ejecución son presentados en la tabla 1 . Los alumnos de todos los grupos de tratamiento mejoraron su ejecución técnica en todas las habilidades (1-20\%). Estas mejoras fueron significativas en la habilidad de saque en el grupo " $M C$ " y en el grupo “ $M E D$ ”. 
Tabla 1. Ejecución a nivel cualitativo (técnica) de las distintas habilidades y tipos de organización de la clase (valores expresados en porcentajes -cumplimiento de objetivos aprendizaje-).

\begin{tabular}{|c|c|c|c|c|c|c|c|}
\hline Habilidad & Planteamiento & $\frac{\text { Pre }}{M}$ & $\frac{\text { Post }}{M}$ & $F$ & $P$ & $\mathrm{~h}^{2}$ & Post Hoc Bonferroni \\
\hline \multirow{3}{*}{ Dedos } & MT & 38.75 & 60.00 & 3.374 & .082 & .415 & - \\
\hline & $\mathrm{MC}$ & 48.75 & 52.50 & .093 & .763 & .160 & - \\
\hline & MED & 41.00 & 52.50 & 2.470 & .133 & .320 & - \\
\hline \multirow{3}{*}{ Antebrazos } & MT & 26.67 & 35.00 & 2.177 & .154 & .292 & - \\
\hline & MC & 31.88 & 32.50 & .016 & .899 & .152 & - \\
\hline & MED & 24.55 & 30.91 & 2.328 & .141 & .309 & - \\
\hline \multirow{3}{*}{ Saque } & MT & 23.75 & 27.50 & .674 & .422 & .122 & - \\
\hline & $\mathrm{MC}$ & 27.14 & 34.29 & 4.281 & .048 & .601 & Post $>$ Pre \\
\hline & MED & 25.00 & 31.00 & 4.315 & .049 & .796 & Post $>$ Pre \\
\hline
\end{tabular}

Los resultados de las pruebas de control cuantitativo de la ejecución son presentados en la tabla 2. Los resultados encontrados presentan variaciones tanto en habilidad como en el gru- po de tratamiento. Las diferencias entre pre-test y post-test no fueron significativas en ningún de los grupos de estudio.

Tabla 2. Ejecución a nivel cuantitativo de las distintas habilidades y tipos de organización de la clase (datos expresados en escala de 0 a 5).

\begin{tabular}{|c|c|c|c|c|c|c|c|}
\hline Habilidad & Planteamiento & $\begin{array}{c}\text { Pre } \\
M\end{array}$ & $\begin{array}{c}\text { Post } \\
M\end{array}$ & $F$ & $P$ & $\mathrm{~h}^{2}$ & Post Hoc Bonferroni \\
\hline \multirow{3}{*}{ Dedos } & MT & 2.08 & 1.77 & 8.087 & .897 & .152 & - \\
\hline & $\mathrm{MC}$ & 1.59 & 1.66 & .422 & .519 & .125 & - \\
\hline & MED & 1.18 & 1.47 & .071 & .897 & .152 & - \\
\hline \multirow{3}{*}{ Antebrazos } & MT & 1.12 & 1.23 & .134 & .716 & .165 & - \\
\hline & $\mathrm{MC}$ & 1.35 & 1.47 & .102 & .751 & .061 & - \\
\hline & MED & 1.12 & 1.00 & .102 & .751 & .161 & - \\
\hline \multirow{3}{*}{ Saque } & MT & 1.29 & 1.46 & .280 & .599 & .181 & - \\
\hline & $\mathrm{MC}$ & 1.23 & 1.71 & 1.167 & .286 & .185 & - \\
\hline & MED & 0.71 & 1.18 & 1.524 & .224 & .227 & - \\
\hline
\end{tabular}

\section{Conocimiento}

Todos los grupos mejoraron su conocimiento conceptual sobre el deporte tras la realización de la unidad didáctica (8-

Tabla 3. Rendimiento del alumno en las pruebas de conocimiento.

\begin{tabular}{lcccccc}
\hline \multirow{2}{*}{ Planteamiento } & Pre & Post & \multirow{2}{*}{$F$} & $P$ & $\mathrm{~h}^{2}$ & Post Hoc Bonferroni \\
\cline { 2 - 3 } & $M$ & $M$ & & & & Post $>$ Pre \\
MT & 46.00 & 57.33 & 6.821 & .012 & .724 & - \\
MC & 54.38 & 62.50 & 3.739 & .059 & .623 & .013 \\
MED & 47.65 & 58.24 & 6.747 & .720 & Post $>$ Pre \\
\hline
\end{tabular}

\section{Calidad y cantidad de práctica}

El "MC" fue en el que se obtuvo un mayor número de repeticiones $\left(F_{2,36}=5.565, \mathrm{~h}^{2}=.897, p=.008\right)$ por parte de los
$11 \%$ de mejora). Estas mejoras fueron significativas para el " $M T$ " y para el " $M E D$ ", pero no para el " $M C$ ". 
presentó menor calidad de ejecución en las repeticiones, en los gestos de dedos y de antebrazos ( $40 \%$ calidad). En el gesto de saque, los tres tratamientos consiguieron valores similares de eficacia (aprox. 40\%). El "MT" y el "MED" presentaron valores similares tanto a nivel de cantidad de práctica como a nivel calidad de practica (10 repeticiones con $45 \%$ de calidad y siete repeticiones con un $43 \%$, respectivamente).

\section{Percepción del alumno}

Los alumnos del "MT" fueron los que presentaron valores significativamente mayores a nivel de percepción de aprendizaje $(\mathrm{X}=63.9 \%, \mathrm{~F} 2,18=17.748, \mathrm{~h} 2=.664, \mathrm{p}=.000)$ y de diversión $(\mathrm{X}=62.0 \%, \mathrm{~F} 2,18=8.314, \mathrm{~h} 2=.480, \mathrm{p}=.003)$ que el resto de modelos de enseñanza (24-23\% de diferencia a nivel de aprendizaje y $12-9 \%$ de diferencia a nivel de diversión). El "MC" y el "MED" presentaron valores similares, no obstante, ambos grupos pese a presentar medias similares presentaron tendencias distintas. El "MC" presentó valores más bajos conforme pasaban las sesiones, mientras que el grupo "MED" presentó valores más altos conforme pasaban las sesiones.

\section{Percepción del profesor}

A partir del análisis de la entrevista realizada a la profesora, se obtuvieron las valoraciones de su percepción del proceso llevado a cabo. Sus opiniones han sido agrupadas en: a) aprendizaje del alumno en función del modelo de enseñanza utilizado; b) interés, autonomía, e implicación del alumno; y c) valoración global del profesor.

a) Aprendizaje del alumno en función del modelo de enseñanza. Con la utilización del "MT", el proceso de aprendizaje estuvo controlado y la profesora podía ver cómo se producía.

"El proceso ha estado controlado y organizado en todo momento" "Llevar la clase es fácil para el profesor. El proceso está muy estructurado. Primero la explicación y ejemplos, después la práctica. Al cabo de unas sesiones, cuando los niños demandan jugar, se les introduce el juego".

Con el "MC", la sensación percibida es que no hubo aprendizaje de los contenidos buscados.

"Se ha notado perfectamente los alumnos que han tenido experiencias previas o que son motrizmente más hábiles" "Los que no tenían esa experiencia o habilidad, no han evolucionado igual y en algunos casos su experiencia no ha sido positiva" "El nińo no asocia jugar con aprendizaje, al final si no le sale, le da igual y no se esfuerza en mejorar o sigue jugando como si nada o evita el balón". "Al final de la unidad didáctica, da la sensación de que los alumnos no han aprendido nada"

En el "MED", la profesora ha percibido que el aprendizaje era sobre aspectos actitudinales y de trabajo colectivo, y no tanto de aspectos procedimentales relacionados directamente con el voleibol.
"Han aprendido a estructurarse, las funciones marcadas, etc." "Respetaban los ejercicios, tiempos de los mismos..." "Su comportamiento ha sido muy bueno" "El grupo parecía haber madurado de repente" "Quizás no haya sido el grupo que más haya mejorado, pero a nivel colectivo si se comportaban como un equipo"

b) Interés, autonomía, e implicación del alumno. Con la utilización del "MT", la profesora percibe la necesidad de que el trabajo sea progresivo para que el alumno perciba su mejoría y se implique en el proceso.

"El alumno ha percibido su mejora" "El voleibol es un deporte muy técnico que requiere de un control del gesto para poder ser jugado" "Si no se percibe la mejora, disminuye la motivación por la práctica" "Con unidades didácticas largas los alumnos se cansan" "Es necesario combinar las tareas con su aplicación de forma progresiva"

El "MC" supuso en los alumnos una implicación al principio que sin embargo cayo notablemente a lo largo del resto de las sesiones.

"Los alumnos del MC, al principio, dos primeras sesiones, eran el mejor grupo, pero pasado ese tiempo la implicación del alumno baja mucho en el proceso de aprendizaje se dedicaban a jugar" "Se reduce mucho el interés del alumno en cómo hacen las cosas. Se centra únicamente en el juego" "Hay mayor implicación física... mayor participación de los alumnos a nivel físico en clase"

En el "MED" fue en el cual la profesora percibió mayor implicación y autonomía por parte de los alumnos en el proceso.

"Los alumnos están más contentos con el hecho de que ellos marcasen las pautas y se organizaran" "Es cierto que se disminuye el tiempo de práctica, pero se incrementa notablemente la implicación del alumno" "En algunas ocasiones he tenido que auto-controlarme para no intervenir, no estoy acostumbrada a que la clase sea llevada por ellos"

c) Valoración del profesor. La utilización del "MT" implicó mayor control del proceso, organización del proceso, y menor trabajo por parte del profesor.

"...la clase estaba en todo momento controlada" "(los alumnos) sabían de forma clara cuando empezaba los ejercicios, cuando acaban, que había que hacer" "más fácil de preparar y dirigir (...), o al menos yo estoy acostumbrada a este planteamiento" "la realización al final de la unidad didáctica de ejercicios donde jugaban hizo que valoran lo que habían aprendido"

En el "MC" implica, tras las primeras sesiones, que el profesor tenga que encargarse del control de la clase.

"La clase se descontrola... los alumnos no asocian jugar con aprender, no se preocupan de hacer los gestos" "al final hacen lo que quieren, no respetan los criterios marcados" "... te conviertes en un policía”

En el "MED” sorprendió a la profesora por la implicación 
de los alumnos en el proceso y como estos desarrollaron la sesión. Durante la entrevista, la profesora comento:

"Mi percepción inicial era que el juego iba a ser mejor, pero el sistema con entrenadores me ha sorprendido mucho" "los alumnos se han organizado y realizado las sesiones muy bien" "No creía que fuese capaz este planteamiento con niños de $1^{\circ}$ de ESO. De hecho, cuando me comentáis el estudio, dije que si este grupo iba muy mal a nivel de organización y control que quería tener libertad de cambiar la dinámica de clase (sacarlo del estudio)"

"Es una propuesta muy cómoda, te puedes centrar en los detalles e ir donde haces faltar a ayudar"

\section{Discusión}

El propósito del estudio fue valorar el efecto de tres modelos de enseñanza sobre el proceso de enseñanza-aprendizaje en clases de educación física. Los resultados muestran que en los diferentes planteamientos llevados a cabo se logra un aprendizaje significativo a nivel cualitativo y de conocimiento. El modelo " $M T$ " implicó aprendizaje de las habilidades (ser capaces de realizarlas) a nivel cualitativo y ninguno a nivel cuantitativo, supuso un incremento del conocimiento del contenido (11\%). El " $M C$ " implicó aprendizaje de las habilidades a nivel cualitativo y cuantitativo (ser capaces de realizarlas), supuso un incremento del conocimiento del contenido $(8 \%)$, y la realización de un mayor número de repeticiones de las habilidades, aunque con los niveles de calidad más bajos. El " $M E D$ " implicó una mejora del aprendizaje de las habilidades a nivel cualitativo (sobre todo en el saque), un leve aprendizaje a nivel cuantitativo, y supuso un incremento del conocimiento del contenido (9\%), y la realización de un menor número de repeticiones de las habilidades.

Los resultados anteriores muestran un nivel de aprendizaje bajo en las habilidades a nivel de ejecución técnica y de precisión, independientemente del modelo de enseñanza utilizado. A pesar de ello, cabe destacar que, de los tres gestos trabajados, el pase de dedos, que es más similar con otras habilidades (lanzamiento) fue el que presentó valores de adquisición del $60 \%$ en el pre-test y post test a nivel cualitativo (proceso). Las otras dos habilidades, una nueva y diferente (golpeo de antebrazos) y otra nueva (saque de abajo), presentaron niveles más bajos (<35\%). Estos resultados parecen indicar que, o bien las tareas planteadas no son adecuadas, o bien que el tiempo de práctica empleado no ha sido suficiente como para estabilizar el aprendizaje en estas habilidades. Además, cuanta mayor dificultad y/o novedad implica el gesto técnico practicado, más tiempo parece ser necesario para lograr algún tipo de mejora. En relación a la primera de las posibilidades, indicar que las tareas de los distintos modelos de enseñanza fueron sencillas y básicas (iniciación al deporte), obteniéndose de manuales de iniciación de este deporte (ej. propuesta de abordaje del voleibol a nivel escolar del MEC - Santos, Delgado, y Viciana, 1996). Además, la profesora era experta en el deporte (entrenadora nacional), y entendió que las tareas eran de baja dificultad. En relación a la calidad de la práctica, los resultados muestran la necesidad de replantearse algunas posibles concepciones de los profesores, como, por ejemplo, que reduciendo el tiempo que se dedica a cada uno de los contenidos, y abordando un gran número de aspectos del curriculum de forma más superficial, se va a lograr un mejor aprendizaje y la implicación de los alumnos en el proceso. Estas observaciones irían en la misma línea de las afirmaciones de Sweeting y Rink (1999), en relación a que si no se desarrolla de forma adecuada y en profundidad las habilidades pueden ocurrir que se esté perdiendo el tiempo con los planteamientos realizados. Por tanto, es necesario que el docente establezca el número de sesiones utilizadas y los objetivos de aprendizaje buscados, en función de la dificultad de la habilidad enseñada. Esto puede implicar unidades didácticas de diferente duración (en función de la dificultad del contenido), en detrimento de no poder abarcar todos los objetivos establecidos por el curriculum actual.

Los resultados encontrados a nivel conceptual muestran como con los tres planteamientos metodológicos utilizados se ha logrado una mejora a nivel de conceptos (8-12\%), aunque como se ha indicado en el párrafo anterior, esta mejora de los conceptos no se ha visto aplicada a una mejora en la realización práctica de la habilidad. No obstante, llama la atención que precisamente en el modelo comprensivo esta mejora no sea significativa. Estos modelos de enseñanza se basan en la comprensión, por parte del alumno, de las estructuras y relaciones dadas en el juego, y su posterior aplicación en situaciones de juego de toma de decisiones (González-Villora et al., 2009). En este caso, cabe la posibilidad de que la estructura de las formas jugadas utilizadas no se haya adaptado lo suficiente como para provocar la reflexión en el alumno, que incite un trasvase de cocimiento adquirido de forma práctica al adquirido conceptualmente, y viceversa. Se hace necesario, para próximos trabajos, el revisar la estructura de las situaciones de juego reducidas para este modelo de enseńanza.

En relación al tiempo de práctica desarrollado durante las clases, los resultados encontrados muestran que la propuesta de la utilización de juegos o formas jugadas del MC, con el objetivo de tener más práctica, de forma más aplicada e implicando más al alumno, puede conllevar a que las ejecuciones realizadas no sean correctas. La realización de más repeticiones en situaciones reales implica un coste en la ejecución. Esto pudo implicar una relajación con respecto al cumplimiento de las normas de las actividades y en los criterios de valoración. Se produce, en estos casos, una negociación no consciente entre profesor y alumno, las demandas se reducen con objeto de que se produzca un mínimo esfuerzo por parte de los alumnos en clase (Hastie y Siedentop, 2006). 
Esta "zona de seguridad curricular" hace percibir a los implicados que el proceso funciona de forma adecuada (Rovegno, 1994). Esta percepción produce que este tipo de planteamiento, MC, se perciba como más adecuado que el planteamiento de MT. De hecho, estos planteamientos son denominados como " $M T$ " por parte de sus detractores, a pesar de que los resultados hallados señalan un nivel de práctica y calidad de la misma aceptable. En cualquier caso, el tercer planteamiento abordado ("MED") permite un equilibrio entre número de repeticiones y la calidad de la ejecución. En general, los resultados muestran que los distintos modelos utilizados presentan ventajas e inconvenientes. Serán adecuados si las ventajas se aprovechan y se logra el aprendizaje.

Los resultados respecto a la percepción de los alumnos en el "MT" fue percibido como el más adecuado tanto a nivel de aprendizaje como a nivel de diversión. Estos resultados se pueden deber a varias causas. Por un lado, puede ocurrir que los alumnos estén acostumbrados a este tipo de abordaje metodológico y por eso lo ven cómo más adecuado. También puede ocurrir que los alumnos cuando se inician en una actividad nueva, como era para ellos el voleibol, en ellos se cree incertidumbre, y perciban la necesidad de que las actividades sean dirigidas y controladas por el profesor (Winstein y Schmidt, 1990). En teoría, conforme los alumnos dominen mejor la habilidad van a ser más autónomos, por lo que otras estrategias pueden ser más adecuadas. Otra posibilidad sería la utilización de estrategias específicas para compensar este aspecto (ej. simplificar las tareas o formas jugadas, incrementar la ayuda del profesor, etc.). Estos resultados muestran la necesidad de tener en cuenta la percepción del alumno en el proceso. Esta información facilita que el profesor organice las estructuras de aprendizaje de acuerdo con la evolución del aprendizaje del alumno teniendo en cuenta al alumno, por lo que se le hace partícipe de su progreso. Con esto se podrá cumplir con una de las finalidades buscadas con la enseñanza de la educación física, y es el hecho de que el alumno aprenda de forma autónoma.

La percepción de la profesora sobre el efecto de los modelos aplicados en el aprendizaje de los alumnos, va en la misma línea que la percepción de los alumnos. Cuando el planteamiento se hace de forma estructurada y organizada, el alumno percibe que aprende y, por lo tanto, se encuentra motivado por la práctica. Para el profesor, es más fácil implementar el MT porque implica más organización y control de todo el proceso. La profesora muestra su sorpresa por los problemas de control y sensación de ausencia de aprendizaje de los alumnos acaecidos en el MC. Los alumnos se han centrado en jugar y no en aprender el deporte. El hecho de que hubiese una evaluación externa y, por tanto, una exigencia de cumplir con la realización de unos ejercicios ha podido afectar en el establecimiento de "zonas de seguridad" entre profesor y alumnos (Hastie y Siedentop, 2006; Rovegno,
1994). Con respecto al MED, la profesora indica que, sobre todo, los alumnos han aprendido contenidos a nivel actitudinal, destacando la madurez alcanzada por el grupo y el trabajo en equipo. Estos resultados son importantes si se tiene en cuenta que, entre las competencias a adquirir durante la etapa de educación obligatoria, está entre otras competencias, la de trabajo en equipo y resolución de problemas (LONCE, 2013). Estas mismas reflexiones va en línea con otros trabajos realizados donde se ha aplicado este modelo de enseńanza en educación primaria (Calderon, Hastie y Martinez, 2010); en escolares con adolescentes de 14 y 18 ańos (Hastie y Sinelkinov, 2006, Hastie, Sinelnikov, y Guarino, 2009). Estos trabajos coinciden con la opinión de la profesora en destacar el grado de libertad que permite este modelo de enseńanza, y la posibilidad que da para atender a aquellos alumnos que más lo necesitan dentro del grupo.

En relación a la cesión de responsabilidades al alumno, la profesora indicó su sorpresa con el MED. Sus impresiones muestran que los alumnos son más responsables, organizados, autónomos, etc., si bien, el tiempo destinado a hacer estas tareas reduce sus niveles de práctica y con ello de aprendizaje procedimental. Esto probablemente implica que este tipo de planteamiento requeriría de más tiempo en su implementación para que los alumnos adquieran la dinámica organizativa, y cada vez se aproveche mejor el tiempo de práctica en clase. La profesora valoró de forma muy positiva que este planteamiento permita que los alumnos trabajen y aprendan a funcionar como grupo de forma autónoma. Este planteamiento presenta, según ella nuevas posibilidades para el profesor cambiando el rol del profesor en la clase que pasa de ser director a ser promotor y guía del proceso para llevar a estos a la autonomía en la educación física. Esta reflexión de la profesora va en línea con las conclusiones que se ha llegado en otros trabajos donde también se ha aplicado este modelo de enseñanza (Calderón et al., 2010; McCaughtry et al., 2004; Sinelnikov y Hastie, 2010). No obstante, sería necesario en experiencias futuras, el poder comprobar estos resultados aplicando un instrumento específico que mida la autonomía (Núñez, Martín-Albo, Navarro, y González, 2006), la motivación (Baena-Extremera et al., 2012); y responsabilidad de los alumnos (Escartí, Gutiérrez y Pascual, 2011).

\section{Conclusiones y limitaciones del estudio}

Ninguno de los modelos de enseńanza aplicados mejora de forma significativa el aprendizaje de las habilidades desarrolladas durante la unidad didáctica (pase de dedos, pase de antebrazos y saque), desde el punto de vista cuantitativo. A nivel cualitativo, fue mejor el aprendizaje en los grupos en los que se aplicó el "MC". El "MED" y "MT" proporcionó mejores valores de aprendizaje de conocimientos, que el "MC".

$\mathrm{El}$ "MC" es el que proporciona una mayor cantidad de 
práctica, pero con peores niveles de eficacia, mientras que el "MED" y" MT" proporcionan un nivel más equilibrado entre la cantidad de práctica y la eficacia de la misma. La habilidad del saque fue la que obtuvo los mejores valores de eficacia de ejecución en todos los planteamientos.

Los alumnos percibieron que con el modelo que más aprendieron, y más se divirtieron en clase fue el "MT". Tanto alumnos como profesor percibieron que con el "MC" se aprendió menos, y fue menor el control de la clase. La profesora percibió que con el "MED" se mejoraron aspectos actitudinales de los alumnos como el trabajo en equipo y el respecto por las normas.

Los resultados indican que cada planteamiento metodológico presenta ventajas e inconvenientes, por lo que su empleo variará en función de las características y necesidades del grupo con el que el docente se encuentre. En posteriores trabajos se necesitan profundizar más en el efecto que ejerce cada uno de ellos, ajustando mejor el diseńo de las tareas en algunos casos, y aplicando instrumentos específicos que den información de otros aspectos importantes dentro del proceso de enseñanza-aprendizaje como es la motivación del alumno por las clases, su implicación y responsabilidad en el proceso, y su autonomía.

El presente estudio cuenta con varias limitaciones que deben tenerse en cuenta al extraer conclusiones del mismo. Las dos más importantes fueron: la selección de la muestra (representatividad) y el tamaño de la misma (tres cursos de primer curso de educación secundaria). Los autores son conscientes de las mismas. No obstante, se espera que este estudio sea replicado en diferentes comunidades, ciclos, y contenidos para confirmar si lo realizado en las clases de educación física es eficaz, o no, ya que no cumple con los criterios indicado por los estudios existente en la bibliografía internacional (Kirk, 2006).

\section{Referencias bibliográficas}

1. Behar, J. (1993). Sesgos del observador (Capítulo I). En M.T. Anguera (Ed.), Metodología observacional en la investigación psicológica (volumen II) (pp. 27-76). Barcelona: Promociones y publicaciones Universitarias.

2. Baena-Extremera, A., Granero-Gallegos, A, Bracho-Amador, C. y Pérez-Quero, F.J. (2012). Versión española del Sport Satisfaction Instrument (SSI) adaptado a la Educación Física. Revista de Psicodidáctica, 17(2), 377-396. doi:10.1387/RevPsicodidact.4037.

3. Calderón, A., Hastie, P. A., \& Martínez, D. (2010). Aprendiendo a enseñar mediante el Modelo de Educación Deportiva (Sport Education Model). Experiencia inicial en Educación Primaria. REDALYC, 5(15), 169-180.

4. Calderon, A.; Martinez de Ojeda, D., \& Hastie, P.A. (2013). Valoración de alumnado y profesorado de educación física tras la aplicación de dos modelos de enseñanza. Revista Ricyde, IX, 32, 137-153.

5. Escartí, A., Gutiérrez, M. y Pascual, C. (2011). Propiedades psicométricas de la versión española del Cuestionario de Responsabilidad Personal y Social en contextos de educación física. Revista de Psicología del deporte, 20(1), 119-130.

6. García-López, L. M., Gutiérrez-Díaz, D., González-Víllora, S., \& Valero Valenzuela, A. (2012). Cambios en la empatía, la asertividad y las relaciones sociales por la aplicación del modelo de instrucción educación deportiva. Revista de Psicología del Deporte, 2, 321-330.

7. González-Víllora, S., García-López, L. M., Contreras-Jordán, O. R., \& Sánchez-Mora Moreno, D. (2009). El concepto de iniciación deportiva en la actualidad. Retos, 15, 14-20.

8. Evangelio, C.; González-Villora, S; Serra-Olivares, J. y Pastor-Vicedo, JC. (2016). El Modelo de Educación Deportiva en España: una revisión del estado de la cuestión y prospectiva. Revista Cuadernos de Psicología del deporte, 6, 307-324.

9. Griffin, L., \& Butler, JI. (2005). Teaching games for understanding. Champaign, IL: Human Kinetics.

10. Gutiérrez-Díaz, D., García-López, L. M., Chaparro, R., \& Fernández Sánchez, A. J. (2014). Aplicación del modelo de educación deportiva en segundo de Educación Primaria. Percepciones del alumnado y el profesorado. Cuadernos de Psicología del Deporte, 14(2), 131-144

11. Hastie, P.A., \& Siedentop, D. (2006). The classroom ecology paradigm. In D. Kirk, D. Macdonald \& M. O'Sullivan (Eds.). The handbook of physical education. (pp. 214-225). London: Sage.

12. Hastie, P. A., Sinelnikov, O. A., y Guarino, A. J. (2009). The develop- ment of skill and tactical competencies during a season of badminton. European Journal of Sport Science, 9(3), 133-140.

13. Hernández-Hernández, E., \& Palao, JM. (2013). Diseño y validación de un instrumento para evaluar los contenidos conceptuales sobre voleibol en Educación Secundaria Obligatoria. Apunts. Revista de educación física y deportes, 111 (1), 38-52.

14. Kirk, D. (2006). Sport education, critical pedagogy, and learning theory: toward an intrinsic justification for physical education and youth sport. Quest, 58, 255-264.

15. Kirk, D. \& Macdonald, D. (1998). Situated learning in physical education. Journal of Teaching in Physical Education, 17, 376-387.

16. Light, R. (2008). Complex learning theory- its epistemology and its assumptions about learning: implications for physical education. Journal of Teaching in Physical Education, 27, 21-37.

17. Ley Orgánica para la Mejora de la Calidad Educativa (LOMCE). Ley Orgánica 8/2013, 9 de diciembre. Boletín Oficial del Estado, 295, de 10 diciembre de 2013.

18. Martínez de Ojeda, D., Calderón, A., \& Campos, A. (2012). Percepción de aprendizaje y satisfacción en una unidad didáctica integrada mediante el modelo de educación deportiva. REDALYC, 7, 163-172

19. Méndez-Giménez, A., Fernández-Río, J., \& Méndez-Alonso, D. (2015). Modelo de educación deportiva versus modelo tradicional: efectos en la motivación y deportividad. Revista Internacional de Medicina y Ciencias de la Actividad Física y el Deporte, 56.

20. Mitchell, S., Oslin, J. L., \& Griffin, L. (2006). Teaching sport concepts and skills: A tactical games approach (2nd ed.). Champaign, IL: Human Kinetics.

21. Navarro, V. (2004). 40 ańos de propuestas de juegos motores en Educación Física. Comparativa entre las propuestas tradicionales y modernas de libros-manuales de juegos motores. Revista de educación, 340, 787808 .

22. Núñez, J. L., Martín-Albo, J., Navarro, J. G. y González, V. M. (2006). Preliminary validation of a Spanish version of the Sport Motivation Scale. Perceptual and Motor Skills, 102, 919-930. doi: 10.2466/ PMS.102.3.919-930.

23. Palao, JM. \& Hernández, E. (2007) Manual para la iniciación al voleibol. Murcia: Diego Marín.

24. Palao, JM. \& Hernández, E. (2009). Manual para la iniciación al voleibol. Manual de prácticas. Murcia: Diego Marín. 
25. Rovegno, I. (1994). Teaching within a curricular zone of safety: School culture and the situated nature of student teachers' pedagogical content knowledge. Research Quarterly for Exercise \& Sport, Atlanta, 65(3), 269279.

26. Santos, JA., Viciana, J, \& Delgado, MA. (1996). Voleibol. Madrid: Ministerio de Educación y Ciencia.

27. Serra-Olivares, J. (2014). Conocimiento sobre la táctica deportiva de alumnos de educación primaria. SPORT TK. Revista EuroAmericana de Ciencias de deporte, 3 (1-2), 11-20.

28. Siedentop, D., Hastie, PA., \& Van Der Mars, H. (2011). Complete guide to sport education 2nd. Champaign, IL: Human Kinetics.
29. Sinelnikov, O.A., \& HASTIE, P.A. (2010). A motivational analysis of a season of sport education. Physical Education and Sport Pedagogy, 15(1), 55-69. doi: 10.1080/17408980902729362.

30. Sweeting, T. \& Rink, J. (1999). Effects of direction instruction and environmentally designed instruction on the process and product characteristics of a fundamental skill. Journal of Teaching in Physical Education, 18: 216-233.

31. Winstein, C. J. y Schmidt, R. A. (1990). Reduced frequency of knowledge of results enhances motor skill learning. Journal of Experimental Psychology, 16, 677-691. 